\title{
Fatigue of polyamide mooring ropes for floating wind turbines
}

\author{
Yoan Chevillotte ${ }^{1,2^{*}}$, Yann Marco ${ }^{1}$, Peter Davies ${ }^{2,3}$, Guilhem Bles ${ }^{1}$, and Maël Arhant ${ }^{2}$ \\ ${ }^{1}$ ENSTA Bretagne, Institut de Recherche Dupuy de Lômes (iRDL), FRE CNRS 3744, 29200 Brest, France \\ ${ }^{2}$ IFREMER, Marine Structures Laboratory, 29200 Brest, France \\ ${ }^{3}$ France Energies Marines, 29280 Plouzané, France
}

\begin{abstract}
This paper describes a study of the fatigue characterization of polyamide mooring ropes for floating wind turbines. Under some conditions polyester ropes, which are favoured for offshore platform station-keeping, are too stiff for wind turbine moorings, and polyamide may be a suitable alternative. While early studies on fatigue of braided nylon ropes showed very short lifetimes some recent results have indicated that it is possible to significantly enhance lifetime by modifying rope construction and improving fibre coatings [1]. The fatigue results presented here for ropes from a different supplier, confirm this result. In order to develop an accelerated evaluation of the fatigue performance, heat build-up tests have been performed, and promising first results are shown. Finally, the influence of coating is examined by microscopy and yarn-on-yarn tests, in order to improve understanding of the fatigue mechanisms leading to failure.
\end{abstract}

\section{Introduction}

As the development of Marine Energy Converter systems (such as wave energy generators, tidal current turbines and floating wind turbines) starts to flourish, there is a need for mooring lines in shallow water (between 50 and $100 \mathrm{~m}$ ). Since these applications aim at harnessing marine energies, the systems are located on exposed area where dynamic loading are significant. In addition, the inherent moderate to small water depth induce a global stiff behaviour of the mooring system in using standard components as per done for deepwater floating oil platforms [1] (steel chains or polyester rope). The system integrity will be maintained by damping the dynamic loadings, so requiring the use of more stretchable fibre ropes than polyester.

Mainly because of their use in other marine applications, their low price and high breaking strain (up to 20\%) polyamide (Nylon) is a good candidate for these applications. However, studies on braided Nylon ropes in the 1980's [2] have shown relatively poor fatigue performance which provided little incentive for studies on Nylon mooring ropes. Indeed, Nylon single point moorings offshore are typically changed every one or two years. More recently however, Ridge et al. [3] have shown significantly improved fatigue results for twisted nylon ropes, and those authors qualified these ropes as ideal for wave energy convertor moorings. Weller et al [4] also examined a nylon rope which had been tested at sea for 18 months on a wave energy buoy, and showed the importance of loading history and operating conditions on nylon performance.
The idea of using long term mooring lines in this material is relatively new, and there is little knowledge on the durability behaviour of such structures. It is therefore crucial to further investigate the behaviour of polyamide mooring ropes, in order to optimise their utilisation.

The database is still very limited, only the paper by Ridge et al provides long lay length stranded PA rope fatigue results [3]. Thus the deployment of these fibres for permanent mooring is currently impossible due to the lack of data on their long term mechanical properties.

The goals of the present study are therefore to provide a better understanding of the mechanical behaviour and the fatigue damage of these polyamide ropes, in order to help designing floating wind turbine mooring lines. However, fatigue studies are quite long and costly, this is why an evaluation of rapid characterization by heat build-up measurements [5] [6] has been included in the test programme. This kind of test has proven to be efficient on a wide range of materials [7]. These techniques have been developed and successfully applied in recent years to various polymeric materials included polyamide, from single specimens to structural specimens [8]. However, heat build-up characterization has not been used to date on materials such as ropes or cables. Moreover, a specific challenge is to perform the tests in realistic testing conditions, i.e. with over $90 \%$ humidity. One of the objectives of the study presented here is to investigate if the analysis of heat build-up measurements, which works well on dry solid materials under cyclic loading, can be applied to ropes which damage due to internal friction, and which are tested wet. 
Finally, the influence of coating will be assessed by some yarn-on-yarn abrasion tests and microscopy.

\section{Materials and Test methods}

\subsection{Fibres and ropes}

Polyamide 6 (PA6), often described as Nylon 6 yarns and ropes provided by BEXCO have been investigated in this study. The yarns used are made by Nexis fibers with a tex of 188 , with and without coating. The nylon rope samples used in this series of tests were specially manufactured for the research project. The rope is a sixmeter-long (pin to pin) three stranded rope of outer diameter around 15 millimeters for a nominal break load of $75 \mathrm{kN}$. The lay length is similar to polyester subrope constructions for deepwater mooring ropes and imposes splices of more than 2 meters at each end of the rope, leaving a 1.5 -meter central area.

\subsection{Yarn on Yarn tests}

The yarn-on-yarn abrasion tests were performed as described in CI (Cordage Institute) 2009-02P "Performance requirements for marine grade polyester yarn for fiber rope" [9] and ASTM D 6611, "Standard Test Method for Wet and Dry Yarn-on-Yarn Abrasion Resistance" [10]. This test is the standard method for testing inter-yarn abrasion of synthetic fibers and provides a qualitative value of the abrasion resistance. The only difference from the standard test procedure was that tests were performed in natural sea water here.

These tests were carried out at IFREMER with the configuration presented on Figure 1. A yarn is interwrapped with itself (by turning it by 3.5 turns) between three pulleys. At one end of the yarn a weight is attached to apply a constant tension, and at the other end a motor drives the cable back and forth with a $1 \mathrm{~Hz}$ frequency until the yarn breaks due to abrasion in the wrapped region.

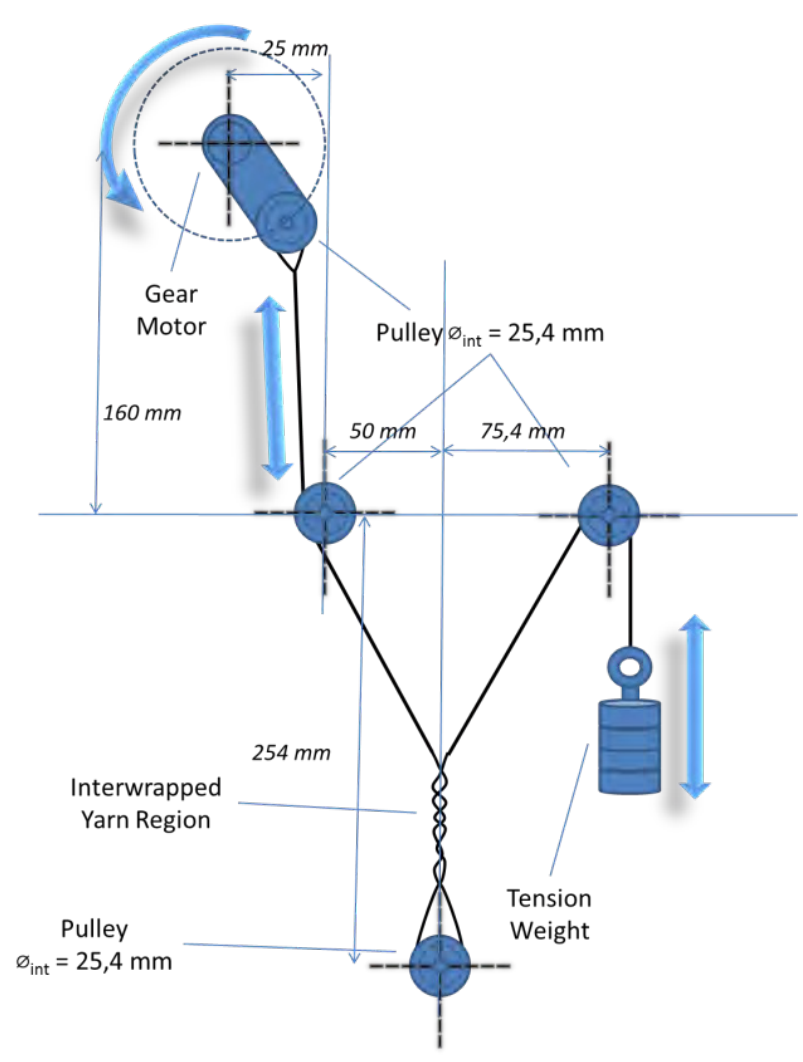

Fig. 1. Yarn on yarn test machine

The inter-wrapped region is kept in sea water throughout the test until failure.

Two types of specimens have been tested with this test: yarn without coating and yarn with a special marine finish.

\subsection{Fatigue tests}

Fatigue testing has been performed on a servo-hydraulic test machine at the IFREMER Marine Structures Laboratory in Brest with a load capacity of $300 \mathrm{kN}$ and a piston stroke of 3 meters.

Before each test the rope sample was immersed in water for 4 hours on the testing machine, and it was then continuously sprinkled with tap water during the test (Figure 2). 


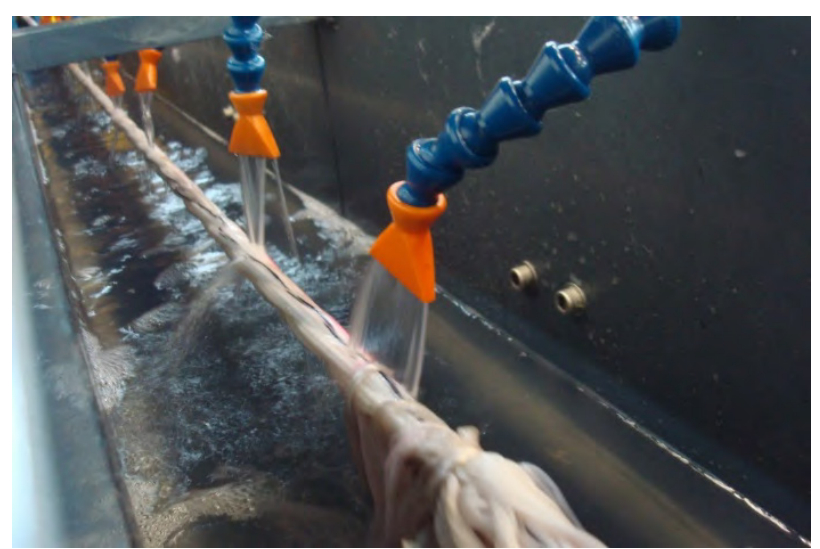

Fig. 2. Rope during a fatigue test

The fatigue test results presented here were performed with a mean-load of $40 \%$ of nominal break load, at a frequency of $0.1 \mathrm{~Hz}$. Before each test, a pre-cycling protocol called "bedding in" was applied. This consists of 5 load-unload cycles from 2 to $50 \%$ of nominal break load.

\subsection{Heat build-up tests}

The heat build-up tests were performed on the same testing device as the fatigue ones. A specific plastic box is used in order to control the reflexion conditions and to simplify the thermal measurements, Figure 3. The temperature was recorded using a Photon $^{\mathrm{TM}}$ camera (FLIR SC7600-BB). The thermal resolution was evaluated around $50 \mathrm{mK}$, which was enough for the protocol applied. The mechanical protocol applied is close to the one applied during the fatigue tests, except that no bedding-in was applied. As the analysis is based on the stabilized response (see below), the transposition of the results obtained from heat build-up tests to the fatigue prediction remains reasonable. Several loading steps with increasing load levels were applied. Each loading step consists of 150 cycles between $2 \mathrm{kN}$ and $\mathrm{XX} \mathrm{kN} \mathrm{(XX} \mathrm{ranging} \mathrm{from} 10$ to 52, see Figure 5).

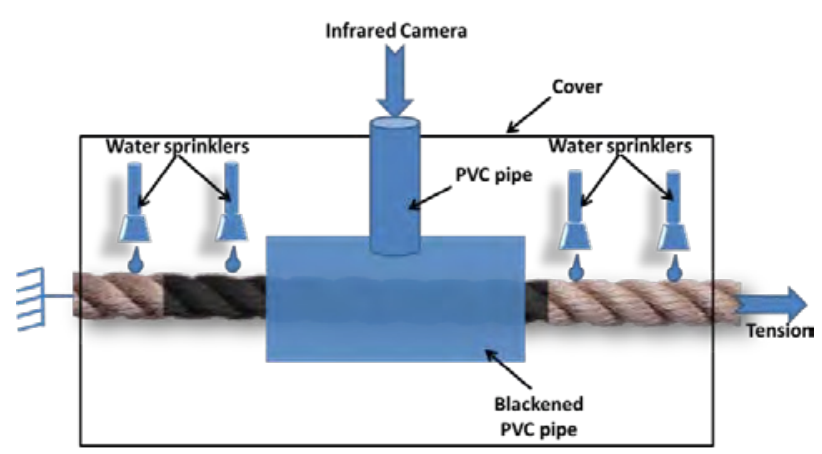

Fig. 3. Scheme of the set-up used for heat build-up tests.

The analysis of the heat build-up tests can be achieved on the evolution of the temperature only, but it is of course much better to investigate the evolution of the dissipated energy, which is an intrinsic parameter. To do so, one can consider either an adiabatic analysis [5] [11] or a stationary analysis [12].

In our study, the stationary state analysis was chosen, in order to catch the thermal response over the full diameter of the ropes. To do so, the thermal evolution is recorded over the full loading steps and averaged over the cable and over the surrounding box (Figure 4). Then these temperatures are combined to compensate the evolution of the ambient temperature during the test. Finally, the relative evolution with time is identified as a saturating exponential function, using the in-house developed Belenos ${ }^{\circledR}$ software [13]. This analytical fit provides the stabilized temperature $\bar{\theta}$ and the characteristic time $\tau_{e q}$. These two parameters are then used to deduce the mean cyclic dissipated energy $\Delta^{*}$, according to Equation (1):

$$
\Delta^{*}=\left(\frac{\rho c \bar{\theta}}{f_{r} \tau_{e q}}\right)
$$

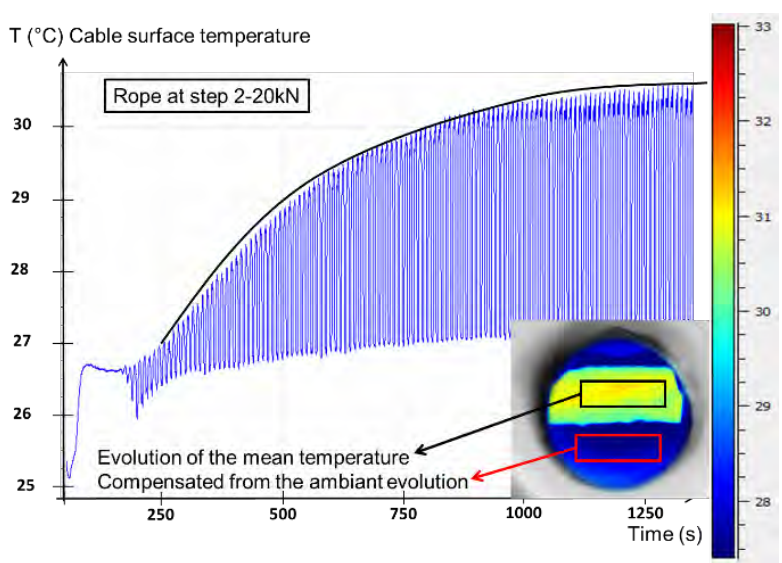

Fig. 4. Typical evolution of the temperature and of the analytical fit.

For each loading step, the mean cyclic dissipated energy $\Delta^{*}$ is evaluated, leading to the construction of the heat build-up curve, relating $\Delta^{*}$ to the maximal force applied (see Figure 5).

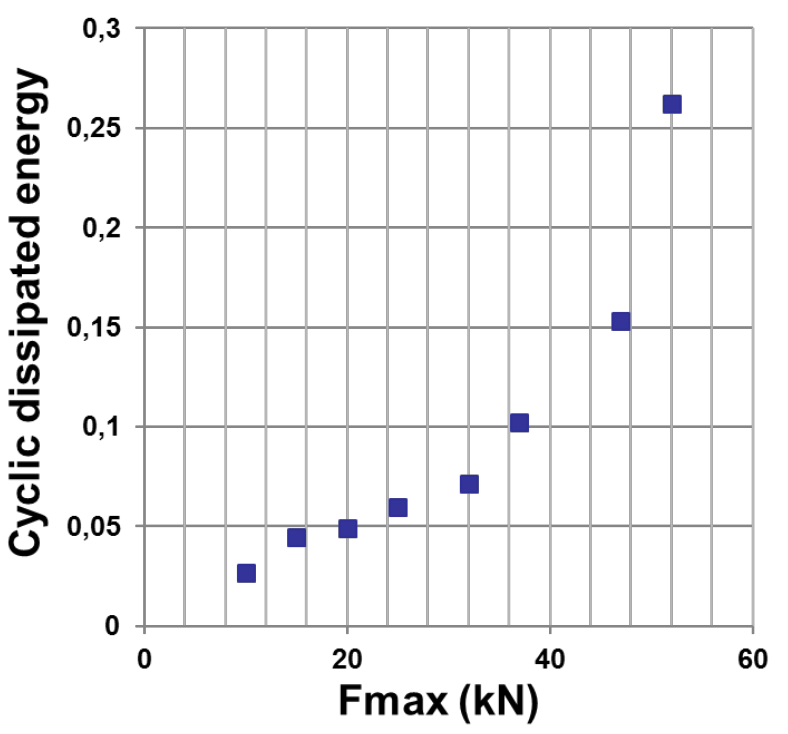

Fig. 5. Heat build-up curve on rope. 


\section{Results and discussion}

\subsection{Yarn on yarn}

Yarn on yarn tests were performed up to failure, which occurred in the inter-wrapped section of the yarn in all cases reported on Figure 6. On the curve, each point is made from 3 to 8 tests depending on the dispersion. The error bars represent the minimum and maximum number of cycles found at this load.

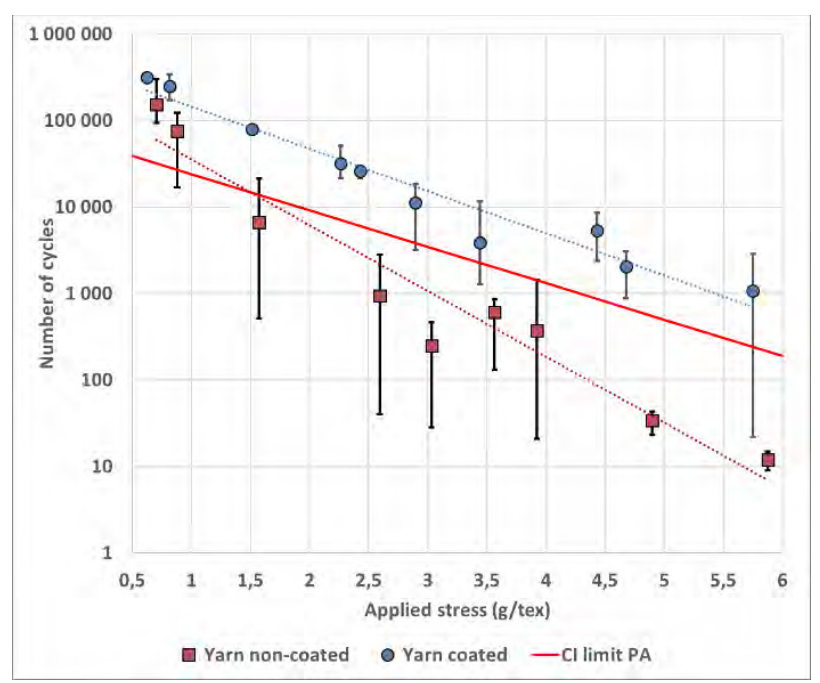

Fig. 6. Yarn on yarn results for uncoated and coated yarns.

Polyamide yarn has a minimum performance criterion (number of cycles versus applied tension) given by the CI document, which is represented as a red line on Figure 6.

The main point of these results is to demonstrate the strong effect of the coating on the abrasion resistance, upgrading the number of cycles to failure by one or two decades, which allow our product to be above the CI limit.

\subsection{Fatigue results}

Fatigue tests were performed up to failure, which occurred in the central section of the rope in all cases. The fatigue data points are shown in Figure 7.

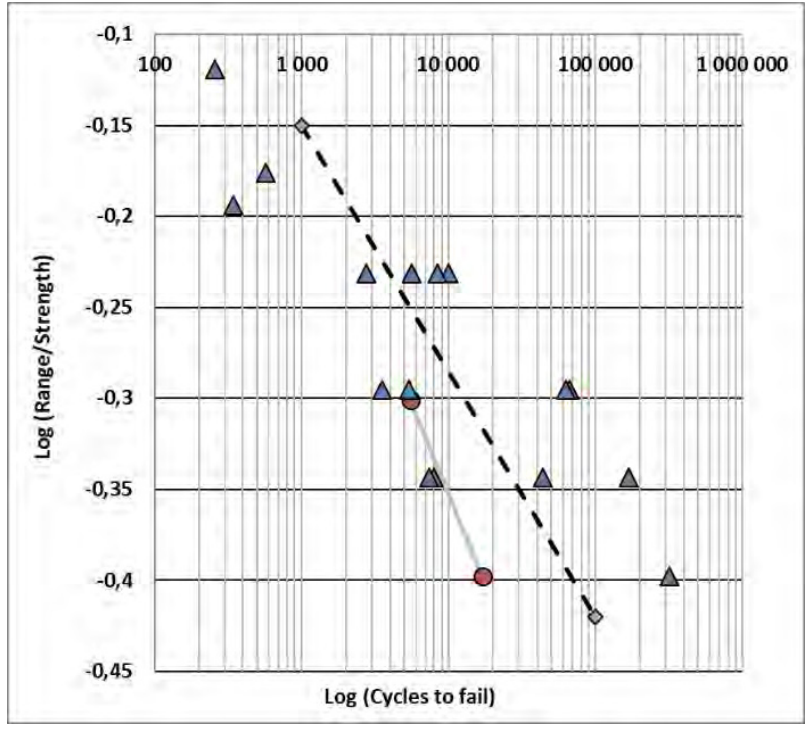

Fig. 7. Example of fatigue test results for rope samples.

Dotted line between the two hexagons shows results from Ridge et al; on their long term mooring polyamide rope for Wave Energy Converters [3]. Our results are fairly close to the one found by Tension Technology International with a different rope manufacturer, and reasonably above the reference curve (grey line between the two red dots) in ISO 18692 (fibre ropes for station keeping) [14] which reinforces the idea that provided appropriate rope constructions and coatings are adopted, nylon fatigue may no longer be an issue for mooring line applications.

\subsection{First analysis of the heat build-up tests and comparison to the fatigue tests}

The analysis of the heat build curve in order to predict the fatigue properties can be more or less complex and accurate, depending on the maturity of the constitutive description [15] or on the quality of the microstructural description [16]. In the case investigated here, these are the very first tests performed on ropes and the analysis will remain very simple. Two features of the heat buildup curve can tentatively be related to the fatigue properties. The first one is the fatigue lifetime obtained for the last loading block that leads to failure (see the top blue arrow on Figure 8). It seems that the lifetime obtained is fully consistent with the fatigue results obtained (see Figure 9). The second one, that is the key point of the heat build-up based analysis, is related to the analysis of the shape of the curve. As explained earlier, the dissipative mechanisms are very numerous (viscoelasticity of the material, friction at several scales in the rope) and an accurate understanding and therefore analysis of the heat build-up curve requires a much deeper investigation. This investigation is underway and should provide a better insight within the next months. 


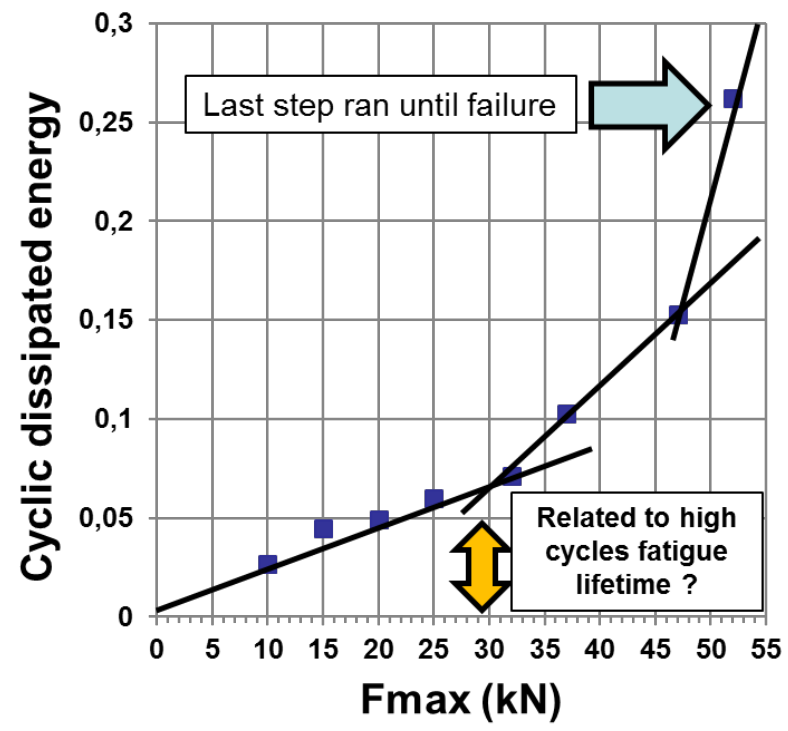

Fig. 8. Illustration of the analysis of the heat build-up curve on rope.

Nevertheless, a rough analysis can be provided by considering that the change of shape of the heat build-up curve could be related to a change of the intensity of the dissipation mechanisms. For several materials [5] [11], this change can be related to the acceleration of the fatigue mechanisms, leading to a very reasonable evaluation of a given fatigue lifetime (usually $10^{6}$ cycles). This evaluation is applied to the curve obtained, as illustrated on Figure 8 (bottom yellow arrow). The force evaluated is also used to plot the predicted lifetime on the same graph as the fatigue tests (see Figure 9). One can conclude that this evaluation, seems also very consistent and that the application of the heat build-up approach to these ropes deserves a closer look.

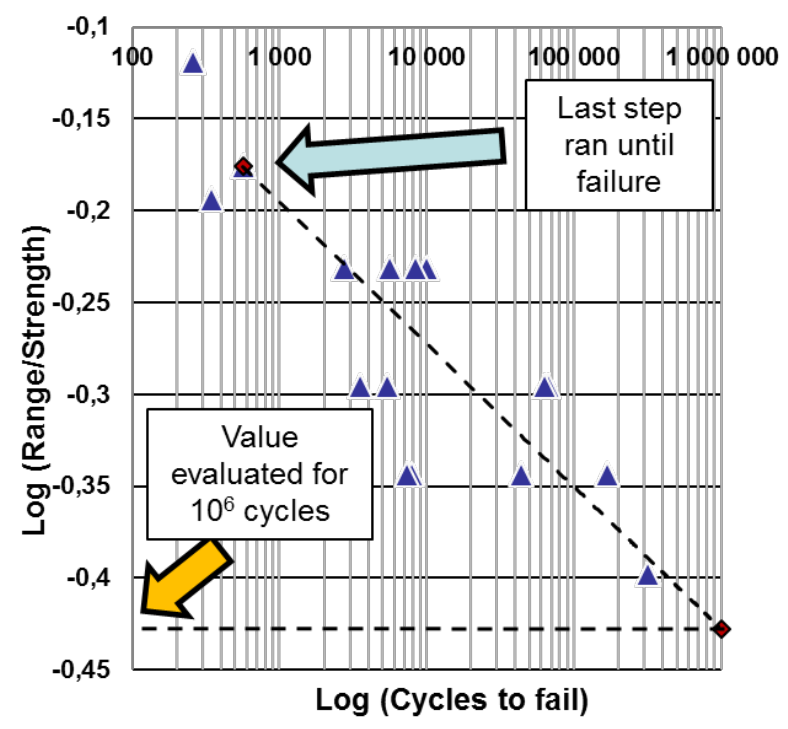

Fig. 9. Comparisons between the fatigue data and the prediction coming from the heat build-up tests.

\subsection{Scanning Electron Microscope SEM}

The aim of this study with SEM (Quanta 200, FEI) analysis was to assess the main phenomena behind fatigue failure and validate if the yarn-on-yarn test samples show the same damage mechanisms as those in rope fatigue testing.

\subsubsection{Rope after fatigue testing}

Samples were taken from broken ropes at $200 \mathrm{~mm}$ from the final failure zone. A significant feature we have found in our observations of broken ropes in fatigue is the presence of melted fibres (Figure 10), but it is not clear if the melting happened during the test or during the breakage of the rope when all the energy was freed. Further observation on samples from tests interrupted before final failure should allow us to determine when the melted zone appears.

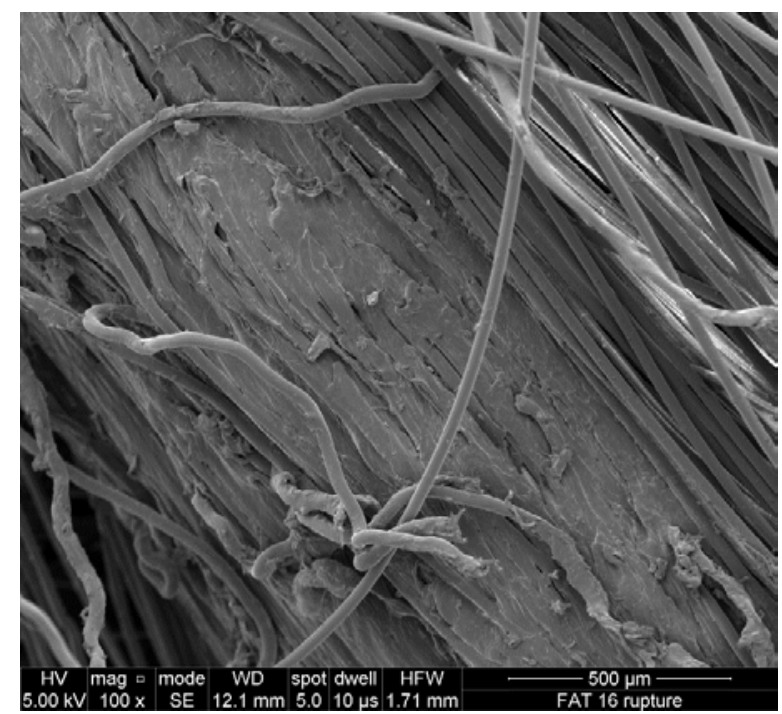

Fig. 10. Example of melted zone in a fatigue rope.

Another feature which seems to be characteristic of the damage developing in this material, is the peel observed on some fibers (Figure 11 and Figure 12), similar behaviour was documented by Hearle in the Atlas of fibre fracture and damage to textiles [17]. 


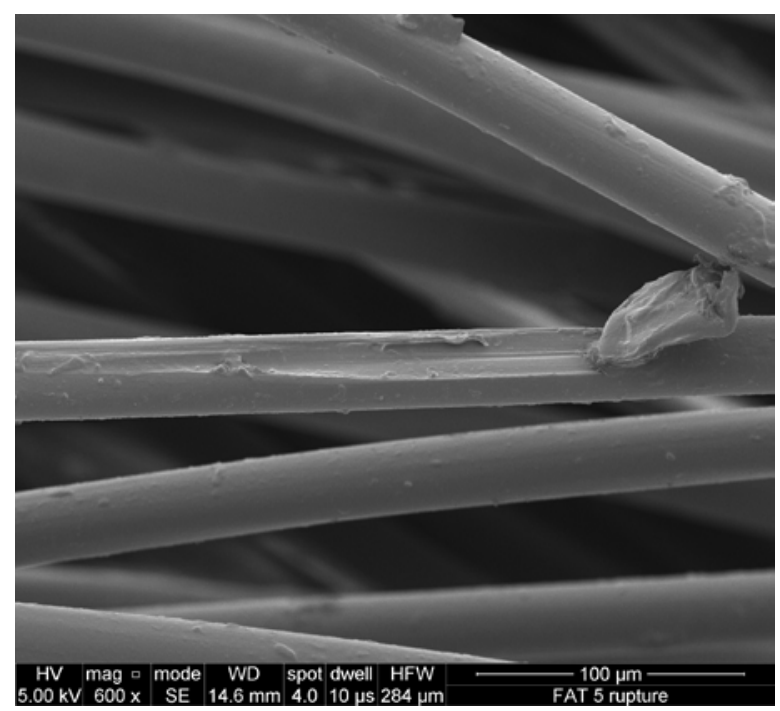

Fig. 11. Example of peeled zone on a fiber after rope fatigue test.

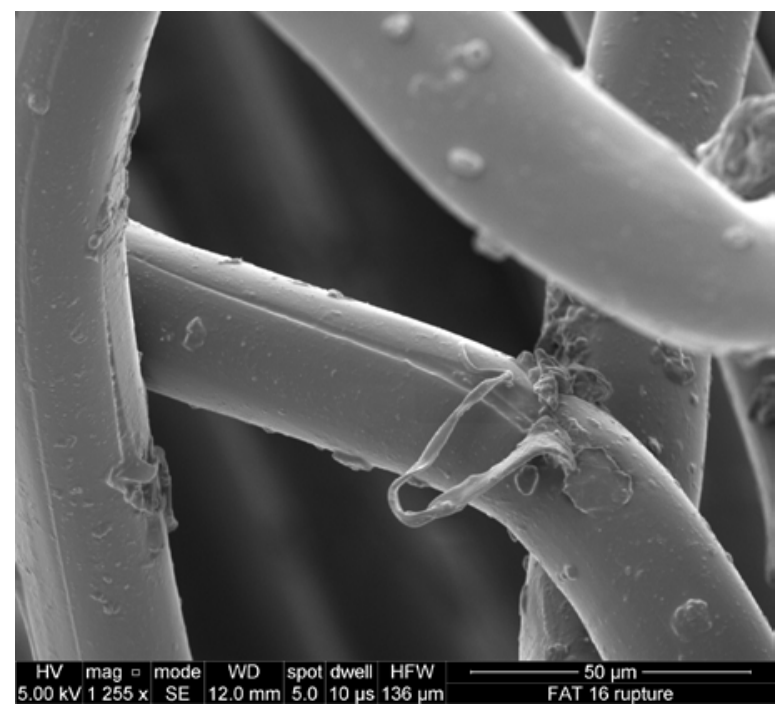

Fig. 12. Example of peeled zone on a fiber after rope fatigue test.

\subsubsection{Yarn samples after Yarn on Yarn abrasion tests}

On specimens after yarn-on-yarn tests, we haven't found melted fibers on specimens observed at low and midrange load. However even at quite low load, we see some marks on broken fibers, which are similar to the peeling seen in the rope fatigue specimens. Mainly, on broken fibers we see either a skin shape where the center of the fiber is gone, Figure 13 or part of the fiber surface is removed like in Figure 14 or damaged like in Figure 15.

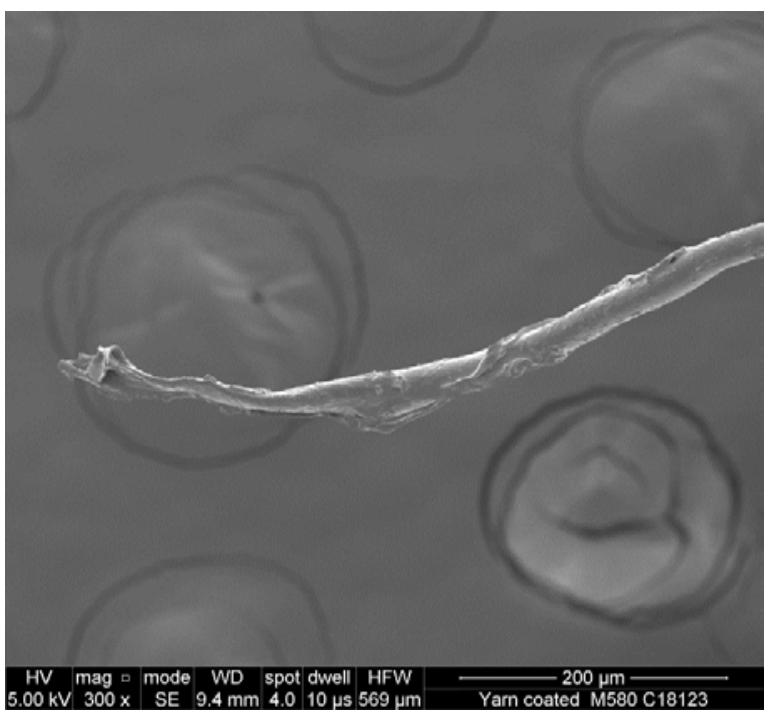

Fig. 13. Example of peeled zone on a fiber after yarn on yarn.

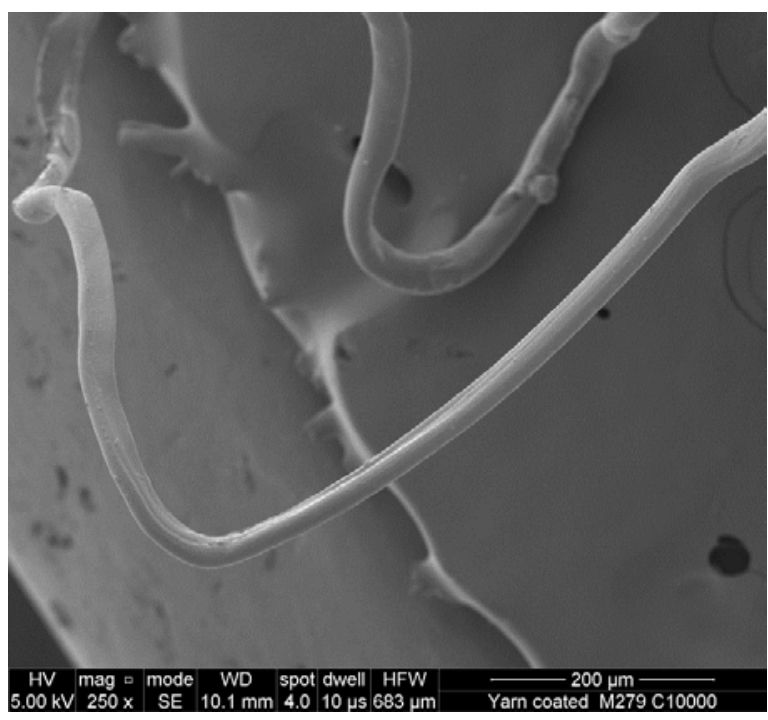

Fig. 14. Example of peeled zone on a fiber after yarn on yarn.

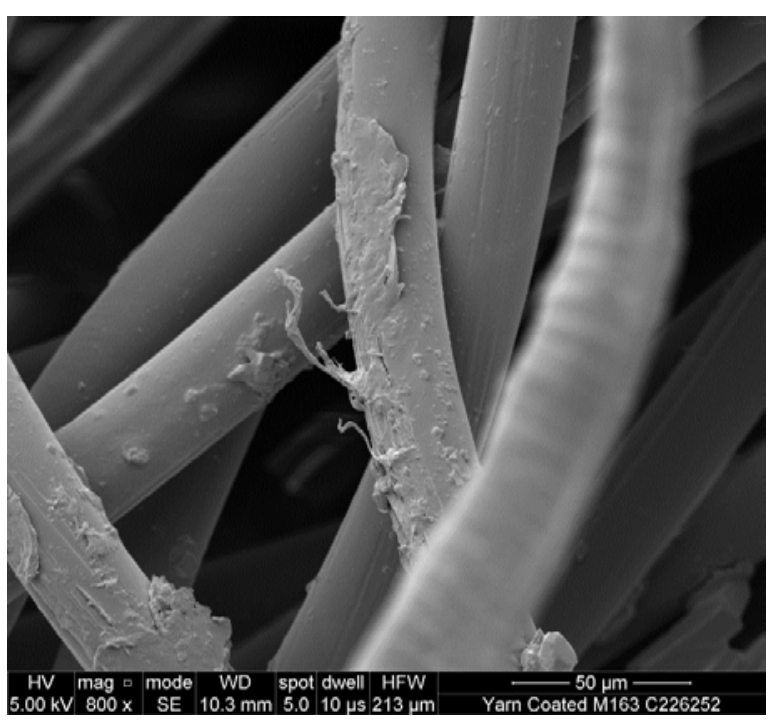

Fig. 15. Example of peeled zone on a fiber after yarn on yarn. 
More tests are underway to examine in more detail these mechanisms.

\section{Conclusion}

The results from abrasion tests by yarn-on-yarn loading show a significant increase in abrasion resistance by the addition of a specially developed coating. That and the long lay length rope construction enables these polyamide ropes to exhibit very good fatigue performance, similar to those demonstrated by Ridge et al.

A first assessment by heat build-up analysis, demonstrates that measurement is possible on a wet rope and shows a promising result which seems consistent with fatigue results even if the loading was not identical. However, an analysis based on temperature alone is not satisfactory because of variations of the heat exchange conditions, which oblige us to adapt the characteristic time. Another campaign of testing will be performed shortly on a different scale of rope to further investigate this aspect.

SEM observations shows two particular failure mechanisms, the first and most visible is melted fibres usually on a large area of the size of a yarn. The second mechanism is the peeling of fibres, this is probably indicative of abrasion in our rope as we found similar behaviour on broken fibres from the yarn on yarn test samples.

\section{Acknowledgements}

This work was performed within the FEM/ANR POLYAMOOR project (ANR-10-IEED-0006-16). This is led by France Energies Marines with partners Naval Energies, Bureau Veritas, Bexco Ropes, Ensta Bretagne and IFREMER.

Thanks also to Nicolas GAYET of IFREMER for SEM photos.

\section{References}

1. Weller, S. D., Johanning, L., Davies, P., \& Banfield, S. J. Synthetic mooring ropes for marine renewable energy applications. Renewable Energy, 83, 12681278. (2015).

2. Kenney, M. C., Mandell, J. F., \& McGarry, F. J. Fatigue behaviour of synthetic fibres, yarns, and ropes. Journal of materials science, 20(6), 20452059. (1985).

3. Ridge, I. M. L., Banfield, S. J., \& Mackay, J. Nylon fibre rope moorings for wave energy converters. In OCEANS 2010 (pp. 1-10). IEEE. (2010, September).

4. Weller, S. D., Davies, P., Vickers, A. W., \& Johanning, L. Synthetic rope responses in the context of load history: The influence of aging. Ocean Engineering, 96, 192-204. (2015).

5. La Rosa, G., \& Risitano, A. Thermographic methodology for rapid determination of the fatigue limit of materials and mechanical components.
International journal of fatigue, 22(1), 65-73. (2000).

6. Le Saux, V., Marco, Y., Calloch, S., Doudard, C., \& Charrier, P. Fast evaluation of the fatigue lifetime of rubber-like materials based on a heat build-up protocol and micro-tomography measurements. International Journal of Fatigue, 32(10), 15821590. (2010).

7. La Rosa, G., and A. Risitano. "Thermographic methodology for rapid determination of the fatigue limit of materials and mechanical components." International journal of fatigue 22.1 (2000): 65-73.

8. Marco, Yann, Vincent Le Saux, L. Jégou, A. Launay, Leonell Serrano, Ida Raoult, and Sylvain Calloch. "Dissipation analysis in SFRP structural samples: Thermomechanical analysis and comparison to numerical simulations." International Journal of Fatigue 67 (2014): 142-150.

9. Flory, J. F. Cordage institute guidelines for marine grade nylon and polyester rope-making yarns. In Oceans-San Diego, 2013 (pp. 1-6). IEEE. (2013, September).

10. ASTM D 6611, "Standard Test Method for Wet and Dry Yarn-on-Yarn Abrasion Resistance, ASTM, West Conshohocken, PA, 2000, re-approved 2007.

11. Marco, Y., Masquelier, I., Le Saux, V., \& Charrier, P. Fast prediction of the wöhler curve from thermal measurements for a wide range of $\mathrm{nr}$ and $\mathrm{sbr}$ compounds. Rubber Chemistry and Technology, 90(3), 487-507. (2017).

12. Jegou, L., Marco, Y., Le Saux, V., \& Calloch, S. Fast prediction of the Wöhler curve from heat buildup measurements on Short Fiber Reinforced Plastic. International Journal of fatigue, 47, 259-267. (2013).

13. V. Le Saux, Belenos users manual, Internal report. ENSTA Bretagne (2017).

14. International Organization for Standardization, "Fibre ropes for offshore station keeping Polyester", Norme ISO 18692, 2007.

15. Ezanno, Anthony, Cédric Doudard, Sylvain Calloch, and Jean-Loup Heuzé. "A new approach to characterizing and modeling the high cycle fatigue properties of cast materials based on self-heating measurements under cyclic loadings." International Journal of Fatigue 47 (2013): 232-243.

16. Marco, Yann, Bertrand Huneau, Isaure Masquelier, Vincent Le Saux, and Pierre Charrier. "Prediction of fatigue properties of natural rubber based on the descriptions of the cracks population and of the dissipated energy." Polymer Testing 59 (2017): 6774.

17. Hearle, J. W., Lomas, B., \& Cooke, W. D. Atlas of fibre fracture and damage to textiles. Elsevier. (1998). 